\section{Rehabilitación del adulto mayor y estilo de vida saludable}

\author{
Rehabilitation of the elderly adult and healthy lifestyle \\ Reabilitação do estilo de vida de idosos adulto e saudável \\ Mariana Ontivero \\ marianaontivero04.05@gmail.com \\ Joelimar Pérez \\ joelimar2018@gmail.com
}

Artículo / Investigación

Article / Research

Conteúdo / Investigação

Recibido septiembre 2017 / Revisión octubre 2017 / Aceptado noviembre 2017

\section{RESUMEN}

Introducción. En la tercera edad, existen temores, preocupaciones y angustias, por lo que es necesario actividades físicas, que permitan optimizar la condición motora y psíquica, y por ende mejorar el estilo de vida. Objetivo. Realizar un Plan de Rehabilitación del Adulto Mayor a través de actividades físicas, que promuevan un estilo de vida saludable, a través de sus potencialidades, en la Casa Hogar "Dios es Amor", Falcón/Venezuela. Materiales y métodos. Se realizó una investigación de campo, bajo un paradigma socio-crítico, con método de la investigación acción-participante, para la transformación social, con la implementación de un Plan, derivado de las diferentes fases de un proyecto factible. Resultados. Implementar ejercicios físicos paulatinamente, que fomenten la participación individual y colectiva, así como desarrollar actividades de divulgación de dicho Plan. Conclusión. La mayoría de los pacientes que serán sometidos al Plan de Rehabilitación, deberían tener una mejoría significativa de salud, por su desempeño individual e independencia; comprobándose en la realización de sus movimientos y no manifestando molestias. De hecho, lo que se persigue es aumentar la autoestima, la vitalidad, autosuficiencia y mejor estilo de vida.

Palabras clave: Rehabilitación; adulto mayor; estilo de vida

\section{ABSTRACT}

Introduction. In the third age, there are fears, worries and anxieties, so it is necessary physical activities, which allow to optimize the motor and psychic condition, and therefore improve the lifestyle. Objective. Carry out a Rehabilitation Plan for the Elderly through physical activities that promote a healthy lifestyle, through their potential, in the "God is Love" Home, Falcón / Venezuela. Materials and methods. A field investigation was carried out, under a socio-critical paradigm, with a participant-action research method, for social transformation, with the implementation of a Plan, derived from the different phases of a feasible project. Results. Implement physical exercises gradually, that encourage individual and collective participation, as well as develop activities to disseminate said Plan. Conclusion. The majority of the patients that will be submitted to the Rehabilitation Plan, should have a significant improvement in health, due to their individual performance and independence; checking in the realization of their movements and not showing discomfort. In fact, what is pursued is to increase self-esteem, vitality, self-sufficiency and better lifestyle.

Keywords: Rehabilitation, elderly, lifestyle
MO: Universidad Nacional Experimental, "Francisco de Miranda", área: Ciencias de la Salud. Programa: fisioterapia. Falcón/Venezuela.

JP: Universidad Nacional Experimental, "Francisco de Miranda", área: Ciencias de la Salud. Programa: fisioterapia. Falcón/Venezuela. 


\section{RESUMO}

MO: Universidad Nacional Experimental, "Francisco de Miranda", área: Ciencias de la Salud. Programa: fisioterapia. Falcón/Venezuela.

JP: Universidad Nacional Experimental, "Francisco de Miranda", área: Ciencias de la Salud. Programa: fisioterapia. Falcón/Venezuela.
Introdução Na terceira idade, há medos, preocupações e ansiedades, sendo necessárias atividades físicas que permitam otimizar a condição motora e psíquica e, consequentemente, melhorar o estilo de vida. Objetivo Realizar um Plano de Reabilitação para os Idosos através de atividades físicas que promovam um estilo de vida saudável, através do seu potencial, no Lar "Deus é Amor", Falcón / Venezuela. Materiais e métodos Foi realizada uma investigação de campo, sob um paradigma sócio-crítico, com um método de pesquisa-ação participante, para transformação social, com a implementação de um Plano, derivado das diferentes fases de um projeto viável. Resultados Implementar exercícios físicos de forma gradual, que estimulem a participação individual e coletiva, bem como desenvolver atividades de divulgação do referido Plano. Conclusão A maioria dos pacientes que serão submetidos ao Plano de Reabilitação, deve ter uma melhora significativa na saúde, devido ao seu desempenho individual e independência; Verificando a realização de seus movimentos e não mostrando desconforto. De fato, o que se busca é aumentar a auto-estima, vitalidade, auto-suficiência e melhor estilo de vida.

Palavras chave: Reabilitação; idoso estilo de vida

\section{INTRODUCCIÓN}

$\mathrm{E}$ n la vejez, los ancianos se cargan de temores, preocupaciones, angustias y mitos, por ello es necesario que realicen actividades físicas, que les permitan mejorar su condición motora y psíquica, la cual se ve afectada por el estilo de vida que lleven. El envejecimiento no es sinónimo de una vida sedentaria y rutinaria, al contrario, envejecer es una etapa llena de vitalidad, si así se lo proponen (1). Desde luego, existen estudios que demuestran que la tercera edad no tiene que ser necesariamente un período, donde exista un deterioro de las capacidades físicas e intelectuales, ya que con motivación, vida activa y productividad, ese deterioro sucede de forma lenta. Sin embargo, en el adulto mayor hay una progresiva pérdida de identificación de la imagen corporal, deterioro cognitivo y descenso de la habilidad motriz; a lo que añade la Organización Mundial de la Salud (2): La inactividad física, es el cuarto factor de riesgo para la mortalidad mundial, correspondiendo a un $6 \%$. Por lo tanto, con el la actividad física, se le propicia las condiciones para desenvolverse en un entorno rico y estimulante, cargados de experiencias de aprendizajes y alcance de logros (3).

De allí, que cabe destacar que los adultos mayores albergados en la Casa Hogar Dios es Amor de Puerto Cumarebo, del sector Cumbres de Alta Vista II, municipio Zamora, estado Falcón/Venezuela, existe una realidad: Se resisten al cambio de estilo de vida, a la adquisición de nuevos hábitos y se niegan al disfrute del lugar donde están. Asimismo, manifestaron sus necesidades y emociones como: Tristeza, ansiedad, soledad y baja autoestima, que a su vez generaron aislamiento social y apatía, dentro del grupo. Sustentado en las falsas creencias, de la denominada tercera edad; sabiendo que pueden experimentar nuevas costumbres favorables, que promuevan un buen estilo de vida y por ende disfrute de una mejor calidad de vida, en esta etapa. Situación que llama la atención y motivó la realización de esta investigación

Ésta investigación contribuyo a la realización de un plan de rehabilitación del adulto mayor, por medio de actividades 
físicas, que promuevan un estilo de vida saludable, a través de sus potencialidades.

Manteniendo como propósito la oportunidad de brindar al adulto mayor, un apoyo socio afectivo de satisfacción y socialización, a través de ejercicios, que mejoraran la condición física de los mismos, ya que una interacción adecuada de ellos, con el medio social, mejora su calidad de vida, supervivencia y además el desarrollar ejercicios en su tiempo libre, los aleja de los temores y preocupaciones. Adema este estudio busca mejorar la calidad de vida del adulto mayor mediante actividades estimulantes y creativas, que puedan generar múltiples beneficios: Potenciar la creatividad, favorecer un funcionamiento psicomotriz adecuado, fomentar el contacto interpersonal e integración social, hacer frente a las limitaciones físicas, contribuir al mantenimiento del ser útil, aumentar el autoestima y ser productivo durante el tiempo libre.

\section{MATERIALES Y MÉTODOS}

S e realizó una investigación de campo, bajo un paradigma socio-crítico, con método de la investigación acciónparticipante, para la transformación social, el cual tiene como objetivo promover las transformaciones sociales y dar respuestas a problemas específicos presentes en el seno de las comunidades, pero con la participación de sus miembros (4). Se realizó la implementación de un Plan de Rehabilitación del adulto mayor a través de actividades físicas, que promuevan un estilo de vida saludable a través de sus potencialidades. Por lo que, el estudio se estructuró en seis fases continúas. Fase I, muestra y comunidad, se detallan los aspectos geográficos, políticos, sociales, económicos y culturales, con énfasis en la realidad actual. Fase II, señala los problemas de salud descritos durante el abordaje comunitario de tal forma que se identifica la realidad prioritaria que conlleva al planteamiento del problema, la razón de realizar el estudio (justificación) y los objetivos de la investigación. Fase III, correspondiente al fundamento teórico, legal y metodológico resaltando los siguientes aspectos: las experiencias previas, el sustento teórico, legal. Asimismo, se describen las técnicas e instrumento utilizados en la recolección y análisis de la información luego de recogida las informaciones, el tipo de investigación, diseño e informantes claves. Fase IV, describe los planes operativos y acciones, presentes en la investigación, la justificación, el plan de trabajo, el cronograma de actividades, el presupuesto de gastos. Fase $\mathbf{V}$, plasma la ejecución de la fase de intervención de forma resumida. Fase VI, destaca la evaluación y reflexión de la investigación en función de la matriz de análisis de información, para luego presentar las consideraciones finales.

Para la recolección de información se usó: la observación directa, se utilizó, la entrevista semiestructurada, aplicada al personal (enfermera y gerontóloga) que atiende los ancianos en la unidad geriátrica lo que facilitó tener un contacto directo con los actores sociales, como informantes claves quienes opinaron del caso de los abuelos y el sistema de vida, que ellos llevan desde que ingresaron a la institución. Los medios utilizados para el acopio y registro de las informaciones y eventos fueron; el guion de entrevista, la cámara fotográfica o de video, el diario de campo; para finalmente realizar una triangulación de datos (cuadro 6).

\section{RESULTADOS}

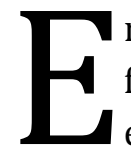
n este apartado se desglosan las 5 fases que constituyo y englobo este estudio mediante el abordaje de cada una, las mismas se describen a continuación: 
Fases I: La Comunidad o escenario donde existe una situación, el mismo cuenta con aspectos tales como: sujetos y la estructura administrativa (3). Se ubicó en la calle Rafael Ocando del sector Cumbres de Alta Vista II, parroquia Puerto Cumarebo, municipio Zamora, Falcón/Venezuela; en la Casa Hogar "Dios es Amor" (3). En entrevista realizada al presidente de la institución, se pudo conocer que la comunidad de Cumbres de Alta Vista II, debe su nombre a la ubicación del sector, en una colina cercana a la urbanización Alta Vista. Esta localidad fue fundada en 1970 por un grupo de 6 familias emigradas de otras comunidades, para mejoras sus condiciones de vida. En el año 1972, estas familias realizaron solicitud a organismos del Estado venezolano (INVISFAL) para la construcción de sus residencias, otorgándose el beneficio a veinte hogares con una población de cuarenta y cinco (45) personas distribuidas en diferentes géneros y edades. Además, cuentan con todos los servicios públicos hasta un ambulatorio tipo II y una cancha deportiva. Asimismo, según la líder comunitaria Sra. Katina Cuauro, la localidad data de unos 30 años de fundada (cuadro 1).

\section{Cuadro 1. Distribución de Población por Género}

\begin{tabular}{|l|c|}
\hline \multicolumn{1}{|c|}{ Caracterización de la población } & $\mathbf{N}^{\circ}$ de Personas \\
\hline Mujeres & 131 \\
\hline Hombres & 168 \\
\hline Total & 299 \\
\hline
\end{tabular}

Fuente: Censo demográfico (2017)

Con respecto, al aspecto cultural, un $75 \%$ de la población profesa la religión católica, sólo una minoría del $25 \%$ es creyente cristiano y existe una iglesia evangélica que realiza actividad comunitaria los domingos, la Danzas Cumarebo. También, Económicamente (5), como el elemento que establece el sistema de vida de una comunidad, a partir de sus potencialidades naturales y modo de subsistencia con relación a la población activa e inactiva. De allí, que se dedican al trabajo agrícola, otros laboran en empresas, instituciones públicas, privadas y particulares que funcionan en el municipio Zamora y Tocópero, desempeñando cargos de: educadores, operadores, vigilantes, carpinteros, herreros, soldadores, topógrafos, enfermeras; otros son trabajadores informales que se dedican al comercio, pescadores, amas de casa que para aportar a los gastos familiares realizan dulcería criolla, costura y artesanía. También, políticamente está organizada jurídicamente con un Consejo Comunal, con sus diferentes mesas técnicas y una contraloría social, comisión electoral, con miembros simpatizantes al partido socialista unido de Venezuela (PSUV), de la unidad democrática. (MUD), independiente $\mathrm{y}$ otros sin tendencia política. Además, socialmente, poseen vías de comunicación, transporte, Radios, red de telefonía fija y móvil a diario.

Fase II: Los problemas de salud, que afectan a la población de adultos mayores, albergados en la unidad geriátrica Casa Hogar "Dios es Amor". Se detectó que 
ameritan un plan de rehabilitación con actividades físicas, que mejoren el estilo de vida. Hecho, que se visualizó a través de 3 encuentros con directivos, estudiantes de fisioterapia, adultos mayores y gerontóloga. Un $75 \%$ presentaba tristeza, dolores articulares, rigidez motora y estilo de vida sedentaria (cuadro 2).

\section{Cuadro 2, Priorización de problemas}

\begin{tabular}{|c|c|c|c|c|c|c|c|c|}
\hline PROBLEMA & A & B & C & D & E & F & TOTAL & PRIORIDAD \\
\hline Dificultad motora gruesa & 2 & 2 & 2 & 2 & 2 & 2 & 12 & I \\
\hline Dolores articulares & 1 & 1 & 1 & 2 & 1 & 1 & 07 & II \\
\hline Sedentarismo & 1 & 1 & 1 & 1 & 1 & 1 & 06 & III \\
\hline
\end{tabular}

Fuente: Gerontóloga de la Casa Hogar “Dios es Amor” (2018)

\section{Leyenda:}

A: Frecuencia: Más-Menos-Repetir

B: Tendencia: Ascendente-Descendente

C: Gravedad: Mayor o Menor

D: Disposición de Recursos. Con factibilidad de acuerdo a los recursos existentes

E: Vulnerabilidad: Más o Menos

F: Frecuencia: $\mathrm{C}$

De allí, que en el envejecimiento no se produce manera en todos los órganos del sujeto (6). Por lo que, la vejez en Latinoamérica se relaciona con una etapa terminal de la existencia del individuo, que implica un retiro de la vida social. Se ve al adulto mayor, como una persona que no puede hacer nada, precedido de prejuicios, limites, normas e inclusive auto encierro, generando en él una especie de aislamiento que en ocasiones no le permite moverse con autonomía (7). Pero, debe considerarse como un estado de bienestar físico, social, emocional, espiritual, intelectual y ocupacional, para realizar actividades físicas, que mejoren su salud y calidad de vida. A lo que se asocia, un cuerpo envejecido es un cuerpo con sensaciones, emociones, sentimientos y necesidades 8). Por lo que se dice, que el envejecimiento, es un proceso complejo y variado, que depende no sólo de causas biológicas, sino de las condiciones sociales de vida y de factores de carácter material y ambiental
(9). También, la CELADE $\quad(10,11)$ determinó que las actividades físicas con ejercicios, tienen la aceptación de las personas mayores por el ejercicio y la recreación, como parte importante para el adecuado mantenimiento de la salud en la sociedad moderna.

Fase III: Fundamentos teóricos, legales y metodológicos. En un estudio que la aplicación de este tipo de un programa de rehabilitación en el adulto mayor genera una actitud positiva en el anciano, la práctica de actividades de esta índole y consecuentemente sacan mejor provecho en el uso de su tiempo libre (12). Igualmente, un estudio en Valencia/ Venezuela, donde se aplicó un Plan de Rehabilitación, demostró que la mayoría de los ancianos les gusta realizar actividad programada y el $90 \%$ de ellos no lo efectuaba de manera habitual 
El $90.9 \%$ manifestó haber mejorado los movimientos de su cuerpo y esto permitía mayor integración grupal, más participación y comunicación entre los compañeros (13). De hecho, la recreación es una práctica sociocultural donde el ser humano en distinta edad hace uso del tiempo libre (14). Por lo tanto, el marco legal del presente estudio se fundamentó en la Carta Magna Bolivariana, en el artículo 84, que expresa: La salud es un derecho social fundamental, obligación del Estado, que lo garantizará como parte del derecho a la vida. El Estado promoverá y desarrollará políticas orientadas a elevar la calidad de vida, el bienestar colectivo y el acceso a los servicios. Todas las personas tienen derecho a la protección de la salud, así como el deber de participar activamente en su promoción y defensa... (15).

Fase V: Planes operativos y acciones: Se representó un esquema detallados de las actividades y tiempo establecido para desarrollar el Plan. Las actividades programadas se proyectan en meses $\mathrm{y}$ semanas (cuadro 3).

\section{Cuadro 3. Cronograma de Actividades}

\begin{tabular}{|c|c|c|c|c|c|c|c|c|c|}
\hline \multirow{3}{*}{ Meses Actividades Año 2018} & \multicolumn{4}{|c|}{ Junio } & \multicolumn{5}{|c|}{ Julio } \\
\hline & \multicolumn{8}{|c|}{ Semanas } & \\
\hline & 1 & 2 & 3 & 4 & 5 & 6 & 7 & & 8 \\
\hline \multicolumn{10}{|l|}{ Visita a la Casa Hogar Dios es Amor } \\
\hline \multicolumn{10}{|l|}{ Evaluación a los abuelos } \\
\hline \multicolumn{10}{|l|}{$\begin{array}{l}\text { Conversatorio con los adultos mayores residentes en } \\
\text { la Casa Hogar Dios es Amor }\end{array}$} \\
\hline \multicolumn{10}{|l|}{$\begin{array}{l}\text { Evaluación médica a los adultos mayores residentes } \\
\text { en la Casa Hogar Dios es Amor }\end{array}$} \\
\hline \multicolumn{10}{|l|}{ Toma de presión arterial a los ancianos } \\
\hline \multicolumn{10}{|l|}{ Calentamiento inicial a los adultos mayores } \\
\hline \multicolumn{10}{|l|}{$\begin{array}{l}\text { Ejercitación al adulto mayor a través de actividades } \\
\text { físicas }\end{array}$} \\
\hline \multicolumn{10}{|l|}{ Conversatorio sobre las actividades realizadas } \\
\hline \multicolumn{10}{|l|}{ Conversatorio con los abuelos. } \\
\hline Entrevista al personal de atención al adulto mayor & & & & & & & & & \\
\hline
\end{tabular}

Fuente: Ontivero/Pérez (2018).

El Plan fue destinados a: Valorar la condición de salud de los adultos mayores participantes en el Plan de Rehabilitación; aplicado a través de actividades físicas, que promovieran un estilo de vida saludable y evaluar el impacto que tiene en la salud (cuadro 4). Asimismo, se realizó un presupuesto (cuadro 5). 
Cuadro 4. Plan de Acción

\begin{tabular}{|c|c|c|c|c|}
\hline $\begin{array}{c}\text { OBJETIVOS } \\
\text { ESPECÍFICOS }\end{array}$ & ACTIVIDADES & $\begin{array}{l}\text { LAPSO DE } \\
\text { EJECUCIÓN }\end{array}$ & RECURSOS & RESPONSABLES \\
\hline $\begin{array}{l}\text { Valorar la condición } \\
\text { de salud de los } \\
\text { adultos mayores } \\
\text { participantes en el } \\
\text { programa } \\
\text { rehabilitación. }\end{array}$ & $\begin{array}{l}\text { Visita a la casa } \\
\text { hogar. } \\
\text { Evaluación a los } \\
\text { adultos mayores. }\end{array}$ & $\begin{array}{c}1 \\
\text { Semanas }\end{array}$ & \multirow{3}{*}{$\begin{array}{l}\text { Humanos } \\
\text { Equipo } \\
\text { investigador } \\
\text { (Estudiantes de } \\
\text { fisioterapia) } \\
\text { - Adultos } \\
\text { mayores. } \\
\text { - Gerontóloga } \\
\text { - Enfermera de } \\
\text { - Directiva da de } \\
\text { la casa hogar. } \\
\\
\text { Materiales } \\
\text { - Guía de díscicios } \\
\text { ejer densiómetro } \\
\text { - Tésicos. } \\
\text { - Equipo hidratación } \\
\text { de cuerpo. }\end{array}$} & \multirow{3}{*}{$\begin{array}{c}\text { Ontivero/Pérez } \\
\text { (2018) }\end{array}$} \\
\hline $\begin{array}{l}\text { Aplicar el programa } \\
\text { de rehabilitación al } \\
\text { adulto mayor a } \\
\text { través de actividades } \\
\text { físicas promoviendo } \\
\text { estilos de vida } \\
\text { saludables. }\end{array}$ & $\begin{array}{l}\text { Acondicionamiento } \\
\text { neuromuscular } \\
\text { - Ejercitación al } \\
\text { adulto mayor a } \\
\text { través de } \\
\text { actividades } \\
\text { físicas. } \\
\text { - Conversatorio } \\
\text { con los adultos } \\
\text { mayores. } \\
\end{array}$ & $\begin{array}{c}4 \\
\text { Semanas }\end{array}$ & & \\
\hline $\begin{array}{l}\text { Evaluar el programa } \\
\text { de rehabilitación al } \\
\text { adulto mayor a } \\
\text { través de actividades } \\
\text { físicas promoviendo } \\
\text { estilos de vida } \\
\text { saludables. }\end{array}$ & $\begin{array}{l}\text { - } \text { Conversatorio } \\
\text { con los adultos } \\
\text { mayores. } \\
\text { - Entrevista al } \\
\text { personal de } \\
\text { atención al adulto } \\
\text { mayor }\end{array}$ & $\begin{array}{c}3 \\
\text { Semana }\end{array}$ & & \\
\hline
\end{tabular}

Fuente: Ontivero/Pérez (2018)

Cuadro 5. Presupuesto de Gastos

\begin{tabular}{|l|l|c|c|}
\hline \multicolumn{1}{|c|}{ Descripción } & Cantidad & Monto & Total \\
\hline Refrigerio & \multicolumn{1}{|c|}{11 personas } & 10.000 & 10.000 \\
\hline Equipo de Hidratación & $\begin{array}{l}4 \text { artículos (gel } \\
\text { antibacterial, } \\
\text { mentol toallas } \\
\text { húmedas, jabón } \\
\text { antiséptico) }\end{array}$ & 3000 & 30.000 \\
\hline & Total & BsS. 40 \\
\hline
\end{tabular}

Fuente: Ontivero/Pérez (2018)

Fase VI: Evaluación y reflexión de la investigación. Esta se dividirá en una fase aeróbica y otra de tonificación muscular, donde lo ideal es que en su ejecución se estimulen las diferentes capacidades, donde intervienen el mayor número de grupos musculares, por medio de coreografías, juegos, circuitos, fortalecimiento. Tanto la intensidad como la duración de estas fases serán en relación a la condición física de nuestros mayores. Se iniciara con una baja intensidad y una corta duración, para ir aumentando ambos aspectos a medida que la condición física vaya mejorando. En este sentido, a pesar de seguir una conexión y continuidad entre todas las tareas, el equipo investigador controlara el esfuerzo físico, la dificultad y complejidad de todas las actividades planteadas; así como la parte motivacional. 
Cuadro 6. Matriz de Análisis de Información

\begin{tabular}{|c|c|c|c|c|}
\hline \multicolumn{5}{|c|}{ Triangulación de técnicas } \\
\hline Categorías & Entrevistados & Observación & Documental & Contrastación \\
\hline $\begin{array}{l}\text { Estilo de } \\
\text { vida } \\
\text { saludable }\end{array}$ & 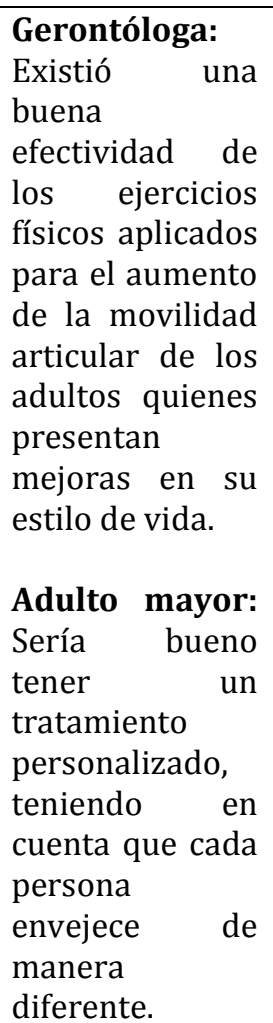 & $\begin{array}{l}\text { Se observan } \\
\text { limitaciones de la } \\
\text { movilidad articular } \\
\text { en los adultos } \\
\text { mayores aquejados } \\
\text { de artrosis de } \\
\text { rodilla, lo que limita } \\
\text { en gran medida su } \\
\text { desenvolvimiento } \\
\text { adecuado en las } \\
\text { tareas de la vida } \\
\text { diaria. }\end{array}$ & $\begin{array}{l}\text { Dosil (2003), } \\
\text { "tener un } \\
\text { estilo de vida } \\
\text { saludable en el } \\
\text { que la } \\
\text { actividad física } \\
\text { desempeñe un } \\
\text { papel } \\
\text { fundamental, } \\
\text { es la mejor } \\
\text { garantía de } \\
\text { obtener } \\
\text { calidad de } \\
\text { vida". }\end{array}$ & $\begin{array}{l}\text { Los adultos } \\
\text { mostraron interés } \\
\text { por mejorar su } \\
\text { estilo de vida, el } \\
\text { ejercicio continuo } \\
\text { permitió } \\
\text { desarrollar tareas } \\
\text { de intensidad } \\
\text { media-baja } \\
\text { que favorecen el } \\
\text { desarrollo de la } \\
\text { resistencia } \\
\text { aeróbica utilizando } \\
\text { el propio } \\
\text { Cuerpo, el espacio } \\
\text { y diferentes } \\
\text { materiales, de } \\
\text { forma continua }\end{array}$ \\
\hline
\end{tabular}

Fuente: Ontivero/Pérez (2018)

\section{Descripción de la actividad}

Al Inicio, se hablara con los abuelos sobre la actividad a realizar, luego habla un calentamiento, con las orientaciones $\mathrm{y}$ modelación de las fisioterapeutas. En el desarrollo, se realizara la flexibilidad del hombro y de la muñeca para ello, los adultos mayores estuvieron en sedestación.

\section{Ejercicios Activos-Asistidos a realizar:}

- Flexión y extensión de hombros 4 series de 10rep.

- Aducción y abducción de hombros 3 series 10rep

- Flexión y extensión de codo y muñeca con una 3 series 10 Rep.

En la culminación de la actividad se efectuará un conversatorio, donde cada uno expresara como se sintió con el ejercicio

Trabajo de flexibilidad: Ejecutar movimiento con diferentes partes del cuerpo a través de ejercicios de estiramiento con el fin de mejorar la elongación muscular, utilizando colchoneta, silla plástica y varilla de madera.

\section{Ejercicios Activos a realizar:}

- Sedestación flexión de cadera por 10seg 3rep

- Sedestación elevación de piernas por 10seg 3rep (Cada una)

- Bipedestación extensión de columna por 10seg 3rep 
- El plan de fisioterapia aplicado a los adultos de la casa hogar Dios es Amor, se basó en:

- Desarrollar actividades de promoción, prevención y rehabilitación biopsicosocial para fomentar, mantener y restaurar la independencia para las actividades de la vida diaria y llevar una vida social activa.

- Establecer en las unidades de primer nivel, una estrategia a través de la cual se puedan detectar la osteoartritis de rodilla en sus primeras etapas y antes de que pueda causar un daño más avanzado de la articulación, ya que cada día se considera una patología de salud pública por la alta frecuencia de casos encontrados en los adultos de edad avanzada.

- Organizar talleres o cursos de actualización de conocimientos y nuevos tratamientos para la capacitación de todo el personal que atiende al adulto mayor en la casa hogar Dios es Amor.

- En relación a la movilidad articular, destacar la necesidad de poner en movimiento las diferentes articulaciones de los adultos para una correcta adaptación a las diferentes tareas a desarrollar, lo cual proporcionara una mejora de la amplitud de todos los movimientos, tanto en modalidad individual y por parejas, para alcanzar lentamente el límite de la articulación y a la vez desarrollar procesos de participación compartida.

\section{CONCLUSIONES}

$\mathrm{L}$ a mayoría de los pacientes que serán sometidos al Plan de Rehabilitación, deberían tener una mejoría significativa de salud, ya que a medida que realicen los ejercicios físicos, su desempeño individual e independencia en las actividades será satisfactorio y positivo; comprobándose en la actitud al realizar sus movimientos y no manifestando molestia. De hecho, lo que se persigue es aumentar la autoestima, que se traduce en vitalidad, autosuficiencia $\mathrm{y}$ mejor estilo de vida.

\section{REFERENCIAS}

1. Fernández $\mathrm{K}$, La recreación en la tercera edad. Tomo (II). Lecturas: Educación física y Deportes. Ediciones Océano. México. 2002.

2. Organización Mundial de la Salud, Estrategia mundial sobre régimen alimentario, actividad física y salud. Consultado en septiembre de 2018. Disponible en https://www.who.int/dietphysicalactivity/pa/es/. 2016.

3. Torres K, Métodos de la Investigación Cualitativa. Ediciones ROMOR. Caracas. Venezuela. 2009

4. Martínez M, (2009) Ciencia y Arte en la metodología Cualitativa. Ediciones Trillas. México.

5. Ponser $\mathrm{P}$, Métodos y Herramientas de la Metodología Cualitativa: Método Investigación Acción Participativa. (10ma Edición) Ediciones Grijalbo. Colombia. 2009.

6. Andrade M, El Adulto Mayor y la Actividad Física. Monografía. UNAH. La Habana. 2006.

7. Sandín E, Investigación cualitativa: Fundamentos y tradiciones. Ediciones Mc Graw Hill. Madrid. España. 2003.

8. Pico W, Cuerpo, dinamismo y vejez. Publicaciones Elmer. Barcelona. España. 2007

9. Febrer V, Calidad de vida, estrés y bienestar. Editorial Psicoeducativa. San Juan, Puerto Rico 2004.

10. Centro Latinoamericano de Demografía Estadística mundial de la Longevidad para los países de Latinoamérica. Volumen.5. $\quad N^{\circ} 1$ Medellín Colombia. 2013.

11. Hernández A, Programa de actividades físicas por medio de ejercicios para el buen uso del tiempo libre en las personas de la tercera edad. Trabajo de Grado para optar al título de Especialista en Cuidado del adulto mayor. Universidad Central de Venezuela. Caracas. 2015 
12. Contreras J, Efectos de un programa de actividades físicas de rehabilitación mediante ejercicios dirigidos a mejorar el estilo de vida de las personas de la tercera edad. Trabajo de Grado para optar al título de Magister en Desarrollo del talento Humano. Universidad de Oriente. Barcelona, estado Anzoátegui. 2015.

13. Urdaneta L, Programa de rehabilitación por medio de ejercicios para proveer estilos de vida saludables. Contexto de estudio: casa hogar "san
Vicente de Paúl" en Naguanagua, estado Carabobo. 2016.

14. Vallejo J, Vivir es Autor realizarse. Reflexiones y Creaciones en Análisis Transaccional (2 $2^{\mathrm{a}}$ ed.). Ediciones Kairo. Barcelona. 2002.

15. Asamblea Nacional Constituyente, Constitución de la República Bolivariana de Venezuela. Gaceta Oficial No. 5.908 de fecha 19/2/2009. Caracas. Venezuela (1999). 